\title{
1,5-Anhydroglucitol in type 2 diabetes mellitus patients with ST elevation myocardial infarction
}

\author{
Dinara Sheryazdanova, Yelena Laryushina \\ Department of Internal Diseases, Karaganda Medical University, Karaganda city, Republic of Kazakhstan
}

Received: 2021-02-02.

Accepted: 2021-06-13

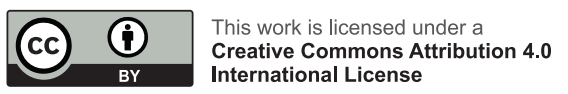

J Clin Med Kaz 2021; 18(4):52-57

Corresponding author:

Dinara Sheryazdanova.

E-mail: sheryazdanova.dinara@gmail.com;

ORCID: 0000-0002-9029-4326

\section{Abstract}

Aim: The study aimed to investigate the potential association of 1,5$A G$ and cardiovascular events in patients with type 2 diabetes mellitus.

Material and methods: The study was an observational nonmatched case-control study. The study included 114 patients with type 2 diabetes mellitus; 42 were with STEMI (STEMI group) and 72 without STEMI (non-STEMI group). Clinical data, fasting blood glucose, HbAlc, and 1,5 Anhydro-D-glucitol were analyzed. The Mann-Whitney U-test and the $\chi 2$ test was used for categorical variables to assess the significance of differences between the groups. The binary logistic regression was used to determine the association of 1,5-AG and STEMI.

Results: There were $43.86 \%$ men and $56.14 \%$ women among the investigated patients. The median age of patients in the STEMI and nonSTEMI groups were 61 (Q25-75 54-71) and 53 (Q25-15 75 57-60) years of age, $\mathrm{p}=0.027$. Median fasting plasma glycemia in the STEMI group was $9.81 \mathrm{mmol} / \mathrm{l}$ (Q25-75 7.7-14.8), in the non-STEMI group (8.55 mmol/l, Q2575 6.72-10.07, $p=0.012$ ). 1,5-AG was significantly lower in the STEMI group than in the non-STEMI (the STEMI group: Me=215.85, Q25-75 186.35-19 280.77; the non-STEMI group Me=314.64, Q25-75 250.83-415.08, p=0.000). No significant difference in concentration of HbAlc was found between the groups. The logistic regression model adjusted on confounders demonstrated that low plasma 1,5-AG concentration increases the odds ratio of STEMI in diabetic patients [OR 3,217 (95\% Cl 2,567-7,732), $\mathrm{p}=$ 0,002].

Conclusion: The association between 1,5-AG and STEMI in patients with T2DM suggests the potential beneficial role of 1,5-AG as a cardiovascular risk marker in patients with type 2 diabetes mellitus.

Key words: diabetes mellitus type 2, 1,5-anhydroglucitol, ST elevation myocardial infarction

\section{Introduction}

About $32.2 \%$ of all type 2 diabetes mellitus patients (T2DM) in the world have cardiovascular diseases. Coronary artery disease and ischemic stroke cause approximately half of all deaths among patients with diabetes [1]. The association between T2DM and cardiovascular disease is beyond doubt. The role of T2DM can be illustrated by the results of such famous studies like the Framingham Study and the Multiple Risk Factor Intervention Study [2]. Diabetes is considered one of the most important independent risk factors of cardiovascular disease. It is independently from additional confounders like age, arterial hypertension, smoking, hypercholesterolemia, and left ventricular hypertrophy associated with cardiovascular diseases [3]. The mortality rate from macrovascular complications in patients with T2DM suggested its decisive importance for the further prognosis of the disease [4].

Fasting glucose, postprandial glucose, and glycosylated hemoglobin (HbA1c) used for glycemic control have several limitations. Clinicians traditionally use glycosylated hemoglobin (HbAlc) measurement as the main instrument to assess carbohydrate metabolism in diabetic patients [5]. However, HbA1c concentration does not reflect all the aspects of carbohydrate metabolism and gives little information about glycemic variability [6]. Some studies reported that glycemic variability may be independent risk factors for cardiovascular diseases in 
patients with diabetes [7]. Studies show that patients with high glycemic variability have episodes of hypoglycemia even if they achieve the target HbAlc and fasting plasma glucose [8]. The study of Suk Chon et al. on evaluation of glycemic variability in well-controlled diabetic patient revealed the presence of glycose variability and postprandial hyperglycemia in patients with target $\mathrm{HbAlc}$, which suggests that it is necessary to evaluate glycemic parameters beyond HbAlc in such patients [9]. Nowadays scientists propose not only the use of $\mathrm{HbA1c}$, which is more summative but also separate the fasting and postprandial glycemia indexes in terms of glycemic control optimization [10].

Another marker of glycemic control, such as postprandial glucose, play an essential role in achieving and maintaining comprehensive glycemic control because it is associated with glycemic variability [11]. One of markers of glycemic control is 1,5-AG (1,5-Anhydro-D-glucitol) that can be used as an indicator of short-term glycemia [12]. Glycemic variability is usually measured by continuous glucose monitoring or by some surrogate markers like 1,5-AG or fructosamine. 1,5-AG was independently associated with coronary revascularization, whereas the more obvious glycemic marker HbA1c did not show a similar relationship. Post-meal hyperglycemia and lower levels of 1,5-AG were found to be important risk factors for adverse clinical events after coronary interventions [13].

Studies have shown that $1,5-\mathrm{AG}$ enters the body mainly from food. The average amount of 1,5-AG consumed is $4.4 \mathrm{mg}$ / day. Its chemical structure with a closed pyran ring provides metabolic stability, the rate of consumption corresponds to the daily rate of excretion. 1,5-AG mainly comes from food. It is well absorbed in the intestines and is distributed to all organs and tissues [14]. Renal reabsorption of $1,5-\mathrm{AG}$ is $99.9 \%$, but there is a process of competitive inhibition of 1,5-AG reabsorption by excretion of excess glucose in the urine (glucosuria). Based on these data, Japanese research groups in their studies have demonstrated a decrease in the concentration of $1,5-\mathrm{AG}$ in serum in patients with hyperglycemia compared to patients in an euglycemic state. Studies have shown that measurements of 1,5AG reflect glycemic status during the previous $48 \mathrm{~h}-2$ weeks $[15,16]$. In addition, a gradual normalization of $1,5-\mathrm{AG}$ values was demonstrated for patients responding to glucose lowering drug therapy [17].

We consider it promising to study the role of $1,5-\mathrm{AG}$ as a marker for assessing the risk of ST elevation myocardial infarction (STEMI) in patients with diabetes mellitus 2. Our study aimed to investigate the potential association of 1,5$\mathrm{AG}$ and cardiovascular events in patients with type 2 diabetes mellitus.

\section{Material and methods}

The study was observational non-matched case-control study. The number of study participants was 114 . All the patients were over 18 years old.

The sample size was calculated by the Kelsey method using EPI info statistical calculator for unmatched casecontrol studies. The percentage of controls was $18 \%$ and of cases $39 \%$. The data was taken from the statistical collection of the population health of the Kazakhstan in 2018. The minimal calculated exposed cases were 38 and unexposed 72 with two-sided confidence level $95 \%$, power $80 \%$, and the ratio of unexposed to exposed cases 2 .

The STEMI group (cases) included 42 patients with T2DM, and ST-elevation myocardial infarction (STEMI) developed less than 24-hours before hospitalization. The blood draws were made on admission to hospital, the interviewing on the third day of hospitalization.

The non-STEMI group (control) included 72 patients with T2DM without STEMI. The participants in this group were preselected among patients with T2DM registered at the polyclinic and interviewed at their usual follow-up visit. Patients were asked to come fasting next day to provide the blood draws. Patients were recruited between April and November 2018 at the Center of Cardiac Surgery and Outpatient Clinic No. 1 in Karaganda. Pregnant women, patients with psychiatric disorders and malignant tumors were excluded from the study. All study participants signed the informed consent. The protocol of the study was approved by the ethic committee of Karaganda Medical University, No. 309, date of approval 19.05.2017.

Arterial blood pressure (BP) of the patients was measured by three consecutive measurements using mechanical tonometer (Microlife BP AG1-10) on both hands with a preliminary rest period of at least 10 minutes, in accordance with the principles of the World Health Organization [18]. The lowest measurements were taken for future calculations. The blood pressure was classified and hypertension graded as follows: Optimal - SBP $<120 \mathrm{mmHg}$ and DBP <80 mmHg; Normal - SBP 120-129 $\mathrm{mmHg}$ and/or DBP 80-84 mmHg; High normal - SBP 130-139 $\mathrm{mmHg}$ and/or DBP 85-89 mmHg Grade 1 - SBP (systolic blood pressure) 140-159 $\mathrm{mmHg}$ and/or DBP (diastolic blood pressure) 90-99 mmHg; Grade 2 - SBP 160-179 mmHg and/or DBP 100$109 \mathrm{mmHg}$; Grade 3 - SBP $\geq 180 \mathrm{mmHg}$ and/or DBP $\geq 110$ $\mathrm{mmHg}$; Isolated systemic hypertension - SBP $\geq 140 \mathrm{mmHg}$ and DBP $<90 \mathrm{mmHg}$.

Body weight and height were measured using a digital stadiometer and scales (TBEC RS-232). The body mass index (BMI) was calculated by weight in kilograms divided by height in meters squared. Overweight state was established at BMI of 25$29.9 \mathrm{~kg} / \mathrm{m}^{2}$, while a BMI $\geq 30 \mathrm{~kg} / \mathrm{m}^{2}$ defined obesity. The classes of obesity were: class I obesity (BMI, 30.0 to $34.9 \mathrm{~kg} / \mathrm{m} 2$ ), class II obesity (BMI, 35.0 to $39.9 \mathrm{~kg} / \mathrm{m} 2$ ), and class III obesity (BMI $\geq 40 \mathrm{~kg} / \mathrm{m} 2$ ) Waist circumference (WC) was measured using an inelastic measuring tape at the midpoint between the lower edge of the last palpable rib and the upper part of the iliac crest. The abdominal obesity was established at waist circumference $>94 \mathrm{~cm}$ in men and $>80 \mathrm{~cm}$ in women. The blood glucose level was measured in capillary blood using a glucometer (Accu Chek active). The diagnosis of T2DM was established at HbA1c level greater than or equal to $6.5 \%$ according to the recommendations of the American Diabetes Association [19].

STEMI was diagnosed taking the electrocardiogram and troponin $\mathrm{T}$ level according to The American College of Cardiology, The American Heart Association, The European Society of Cardiology, and The World Heart Federation joint ECG criteria for STEMI [20].

Parameters of carbohydrate metabolism such as fasting plasma glycemia, HbAlc and 1,5-AG were investigated. HbA1c was detected from capillary blood using reflectometer (Nyco-Card test system). The parameters of lipid profile such as Triglycerides (TG), Total cholesterol (TC), Highdensity lipoprotein (HDL) and Low-density lipoprotein (LDL) cholesterol were detected from blood plasma using the method of selective precipitation with phosphotungstate and magnesium (Automatic Analyzer VitaLine-200). Insulin, C-peptide, glucagon, and glucagon-like peptide-1 was measured by multiplex immunological analysis using XMap technology on Bio Plex 3D. The plasma concentration of 1,5-AG was measured by high performance liquid chromatography with mass-selective mass spectrometry. 


\section{Statistical processing}

Normal distribution of quantitative data was examined using the Kolmogorov-Smirnov test. The qualitative data were performed using the number and percentage. The Mann-Whitney U-test was used for non-parametric continuous variables, $\chi 2$ test was used for categorical variables to assess the significance of differences between the groups. The binary logistic regression was used to determine the association of 1,5-AG and STEMI (the outcome variable). The outcome variable has two categories " 0 " means the presence of STEMI, and " 1 " means absence. The $1,5-\mathrm{AG}$ was present as binary variable: normal or decreased. The cut-off level for dividing 1,5-AG to normal or decreased was $247 \mu \mathrm{mol} / \mathrm{l}$. The cut-off point was established by measuring the values of 1,5-AG in a reference group and taking two standard deviations below the mean. The confounders were age, duration of diabetes, glucose, HbA1C. Statistical analysis was provided on IBM SPSS Statistics software, ver. 22.0. Results were considered as statistically significant at $\mathrm{p}<0.05$.

\section{Results}

The Baseline characteristics of patients $(\mathrm{n}=114)$ presented in Table 1. The proportion of men and women in the study was $43.86 \%$ of men and $56.14 \%$ of women. The median age of patients in the STEMI and non-STEMI groups were 61 (Q25-75 54-71) and $53(\mathrm{Q} 25-75$ 57-60) years, $\mathrm{p}=0.027$. The number of men in the STEMI group was $24(48.00 \%)$ and the non-STEMI group - $26(52.00 \%)$. The number of women in the STEMI group was $18(28.12 \%)$, the non-STEMI group - 46 (71.88\%), $\chi 2=3,559, \mathrm{p}=0,059$.

\section{Table 1}

Baseline characteristics of patients $(n=114)$

\begin{tabular}{|c|c|c|c|}
\hline Variable & $\begin{array}{l}\text { The STEMI group, } \\
\mathrm{n}=42 \\
\mathrm{Me}(\mathrm{Q} 25-75)\end{array}$ & $\begin{array}{l}\text { The non-STEMI } \\
\text { group, } \mathrm{n}=72 \\
\text { Me (Q25-75) }\end{array}$ & $\mathrm{p}$-value \\
\hline Age, years & $61(54-71)$ & $53(57-60)$ & 0.027 \\
\hline Men & $24(48,00 \%)$ & $26(52,00 \%)$ & \multirow{2}{*}{$\begin{array}{l}\chi 2=3,559 \\
p=0,059\end{array}$} \\
\hline Women & $18(28,12 \%)$ & $46(71,88 \%)$ & \\
\hline $\begin{array}{l}\text { Length of } \\
\text { T2DM, years }\end{array}$ & $9.35(5.75-14,25)$ & $9.35(4-10.7)$ & 0.069 \\
\hline $\mathrm{BMI}, \mathrm{kg} / \mathrm{m} 2$ & $31.59(27.04-35.27)$ & $29.66(27,11-33,72)$ & 0.180 \\
\hline WC in men, $\mathrm{cm}$ & $103.10(84.00-103.10)$ & $112(98.50-122,00)$ & 0.090 \\
\hline $\begin{array}{l}\text { WC in women, } \\
\mathrm{cm}\end{array}$ & $103.10(97.00-103.32)$ & $100(88.00-110.00)$ & 0.237 \\
\hline $\begin{array}{l}\text { Systolic BP, } \\
\mathrm{mmHg}\end{array}$ & $140(120-150)$ & $130(120-150)$ & 0.598 \\
\hline \begin{tabular}{|l|} 
Diastolic BP, \\
$\mathrm{mmHg}$
\end{tabular} & $80(80-90)$ & $80(80-90)$ & 0.795 \\
\hline $\mathrm{TC}, \mathrm{mmol} / \mathrm{l}$ & $5.92(4.35-6.57)$ & $5.72(5.01-6.68)$ & 0.986 \\
\hline HDL, mmol/l & $0.92(0.80-1.19)$ & $0.99(0.84-1.13)$ & 0.315 \\
\hline LDL, $\mathrm{mmol} / \mathrm{l}$ & $3.82(3.13-4.97)$ & $3.85(3.25-4.15)$ & 0.768 \\
\hline $\mathrm{TG}, \mathrm{mmol} / \mathrm{l}$ & $1.29(0.89-1.74)$ & $1.52(1.06-2.16)$ & 0.083 \\
\hline $\begin{array}{l}\text { Serum } \\
\text { creatinine }\end{array}$ & $107.54(85.96-129.5)$ & $\begin{array}{l}101.52 \text { (75.99- } \\
115.70)\end{array}$ & 0.053 \\
\hline \multicolumn{4}{|c|}{ Treatment with hypoglycemic drugs } \\
\hline Metformin & & $23(62.2)$ & $43(64.2)$ \\
\hline Sulfomiurea & $9(24.3)$ & & $13(19.4)$ \\
\hline IDPP4 & $1(2,9)$ & $4(5.9)$ & \\
\hline Insulin & & $4(10.8)$ & 7 (11.9) \\
\hline
\end{tabular}

Categorical variables are present as n (\%); non-parametric distributed continuous variables as median (first quartile, third quartile). The MannWhitney U test was used for non-parametric continuous variables, $\chi 2$ test was used for categorical variables.

BMI - body mass index; WC - waist circumference; BP - blood pressure; TC - total cholesterol; HDL - high density lipoproteins; LDL - low density lipoproteins; TG - triglycerides; IDPP4 - Dipeptidyl Peptidase-4 Inhibitors. metabolism.

\begin{tabular}{|l|l|l|l|}
\hline Variable & $\begin{array}{l}\text { The STEMI } \\
\text { group } \\
\text { Me (Q25-75) }\end{array}$ & $\begin{array}{l}\text { The non-STEMI } \\
\text { group } \\
\text { Me (Q25-75) }\end{array}$ & p-value \\
\hline Glucose, mmol/1 & $9.81(7.7-14.8)$ & $\begin{array}{l}8.55(6.72- \\
10.07)\end{array}$ & 0.012 \\
\hline HbA1c, \% & $8.2(7.8-9.05)$ & $7.9(6.92-9.52)$ & 0.237 \\
\hline $1,5-A G$ & $\begin{array}{l}215.85(186.35- \\
280.77)\end{array}$ & $\begin{array}{l}314.64(250.83- \\
415.08)\end{array}$ & 0.000 \\
\hline
\end{tabular}

HbA1c - Glycated hemoglobin; 1,5-AG - 1,5-Anhydroglucitol.

The median BMI in both groups was higher than normal. The BMI in the STEMI group was comparable with the nonSTEMI group $(\mathrm{Me} 1=31.59, \mathrm{Q} 25-75$ 27.04-35.27, Me2 = 29.66, $\mathrm{Q} 25-7527.11-33.72, \mathrm{p}=0.180$ ). No significant difference of WC in the studied groups was found (the STEMI group $\mathrm{Me}=103.10$, Q25-75 97.00-103.32, the non-STEMI group $\mathrm{Me}=100$, Q2575 88.00-110.00, $\mathrm{p}=0.237$ ). Despite that the STEMI group had lower WC, there was no statistically significant differences between the groups (the STEMI group $\mathrm{Me}=103.10$, Q25-75 84.00-103.10, the non-STEMI group $\mathrm{Me}=100$, Q25-75 98.50122.00, $\mathrm{p}=0.090)$.

The median of SBP and DBP was greater than normal in all patients investigated. The median SBP and DBP corresponded to the 1st degree of hypertension in the STEMI group. The median BP corresponded to high normal category in the non-STEMI group according to the classification of the European Society of Cardiology (systolic BP: the STEMI group Me=140, Q2575 120-150, the non-STEMI group $\mathrm{Me}=130$, Q25-75 120-150, $\mathrm{p}=0.598$. Diastolic BP: the STEMI group $\mathrm{Me}=80$, Q25-75 8090, the non-STEMI group $\mathrm{Me}=80$, Q25-75 80-90, $\mathrm{p}=0.795$ ).

All the parameters of lipid profile and serum creatinine showed no differences among the groups. The length of T2DM was longer in patients from the STEMI group (the STEMI group $\mathrm{Me}=12.00$, Q25-75: 3.50-17.00, the non-STEMI group $\mathrm{Me}=7.13$, Q25-75: 3.00-13.00, $\mathrm{p}=0.003$ ).

All the patients received one of 3 groups of oral hypoglycemic drugs: Metformin, Sulfonylurea, Dipeptidyl Peptidase 4 inhibitors (DPP 4 inhibitors), or insulin. There was no difference in the number of patients receiving different therapy between both groups.

The level of fasting glycemia in the STEMI group was increased up to $9.81 \mathrm{mmol} / \mathrm{l}$ (Q25-75 7.7-14.8), which was higher than in the non-STEMI group with a median of 8.55 $\mathrm{mmol} / \mathrm{l}(\mathrm{Q} 25-75$ 6.72-10.07), $\mathrm{p}=0.012$. The level of HbA1c in both groups was higher than normal but with no significant difference between the groups.

The parameters of regulation of carbohydrate metabolism are shown in Table 2. There was significant decrease of 1,5-AG concentration in the patients of the STEMI group (the STEMI group $\mathrm{Me}=215.85$, Q25-75: 186.35-280.77, the non-STEMI group $\mathrm{Me}=314.64$, Q25-75: 250.83-415.08, $\mathrm{p}=0.000$ ).

The model of binary logistic regression with STEMI as an outcome variable and 1,5-AG, age, gender, duration of diabetes, plasma glucose, and $\mathrm{HbA} 1 \mathrm{c}$ as covariates shows that at lower 1,5-AG plasma concentration the odds of STEMI in T2DM patients increases [OR 3,217 (95\% CI 2,567-7,732), $\mathrm{p}=0,002$ ]. Another significant parameter was increased plasma glucose [OR 0.603 (95\%CI $0.441-0.823), \mathrm{p}=0,001$ ] (Table 3). 


\begin{tabular}{|l|l|l|l|l|l|l|}
\hline Variable & B & Mean square error & p-value & Exp (B) & \multicolumn{3}{l|}{$95 \%$ confidence interval of EXP(B) } \\
\cline { 4 - 7 } & & & & & Lower & Upper \\
\hline Age & $-0,079$ & 0,045 & 0,082 & 0,924 & 0,846 & 1,010 \\
\hline Gender & $-1,123$ & 0,839 & 0,181 & 0,325 & 0,063 & 1,683 \\
\hline $\begin{array}{l}\text { Duration of } \\
\text { diabetes }\end{array}$ & $-0,029$ & 0,069 & 0,672 & 0,971 & 0,847 & 1,113 \\
\hline Plasma glucose & $-0,506$ & 0,159 & 0,001 & 0,603 & 0,441 & 0,823 \\
\hline HbA1c & 0,307 & 0,295 & 0,298 & 1,359 & 0,763 & 2,422 \\
\hline $1,5-$ AG & 2,654 & 0,873 & 0,002 & 3,217 & 2,567 & 7,732 \\
\hline
\end{tabular}

HbA1c - Glycated hemoglobin; 1,5-AG - 1,5-Anhydro-D-glucitol

\section{Discussion}

The results of our study demonstrate significant decline of 1,5-AG concentration in plasma of diabetic patients with STEMI, whereas its concentration in plasma of patients without STEMI was greatly higher. In addition, reduction of $1,5-\mathrm{AG}$ significantly increased the odds of STEMI by approximately 3 times in adjusted logistic regression model with such covariates like age, gender, duration of diabetes, plasma glucose, and HbAlc.

The database search did not reveal any completed studies investigating the association between 1,5-AG and acute myocardial infarction in patients with T2DM. There are studies devoted to the level of 1,5-AG showing its role as a predictor of coronary artery disease (CAD). Ikeda et al provided the study of 523 consecutive patients who underwent their STEMI coronary angiography and reported that 1,5-AG predicts the prevalence of coronary artery disease in nondiabetic adults [21]. The comparison of $\mathrm{HbA} 1 \mathrm{c}$ with $1,5-\mathrm{AG}$ as a CAD predictor provided by Nobutaka Ikeda et al. from National Center for Global Health and Medicine, Japan, suggested the beneficial role of 1,5-AG as superior to $\mathrm{HbAlc}$ in predicting $\mathrm{CAD}$ prevalence; however, both 1,5-AG and HbA1c correlate with CAD complexity calculated by SYNTAX score [22]. Researchers from Kobe University, Japan, have shown that daily glucose fluctuation may influence on the destabilization of coronary plaque and increase the risk of its rupture in patients with CAD pre-treated with lipid-lowering therapy [23]. In our study, dyslipidemia was present in all the diabetic patients; however, the parameters of lipid metabolism were not different in groups with and without STEMI.

In our study both groups of patients have dyslipidemia combined with carbohydrate metabolism disorders could indicate the lack of adequate glycemic and lipid lowering therapy and disease decompensation. Diabetic patients with STEMI have a decrease of 1,5-AG concentration, which indicates the episodes of postprandial hyperglycemia in the last 2-3 weeks and the lack of sufficient compensation for diabetes [23, 24].

The number of studies has demonstrated that 1,5-AG may have clinical significance for evaluating the effectiveness of treatment, reflecting postprandial glycemia in type 2 diabetes mellitus [24, 25]. For example, a prospective cohort study conducted by the Washington University School of Medicine found that fructosamine and glucose showed a tendency to decrease glycemia as early as the second week of monitoring in the study. In contrast, $\mathrm{HbAlc}$ values are virtually unresponsive to therapy until week 4 , and $1,5-A G$ is more sensitive to changes in glycemic levels and glycemic control according to established markers [26].
According to many scientific reports HbAlc concentration in patients with T2DM may predict the cardiovascular events. The meta-analysis of $\mathrm{Hu} \mathrm{Y}$. supports $\mathrm{HbAlc}$ as an appropriate surrogate endpoint for cardiovascular events [27]. Some population cohort studies of patients with T2DM also show that HbA1c above the target was associated with higher risk of cardiovascular events and mortality [28, 29].

The results of our study show that the concentration of HbA1c was above the target in STEMI and non-STEMI groups. However, no significant differences between the groups of patients with and without STEMI were found, that did not allow us to establish the association between $\mathrm{HbAlc}$ and adverse cardiovascular events. Several studies demonstrated the modest predictive role of $\mathrm{HbA} 1 \mathrm{C}$ for coronary artery disease in patients with diabetes. Data of these studies reduce the role of $\mathrm{HbAlc}$ as the prognostic marker of cardiovascular events [30-32]. In the study of Marzen a Dworacka and Hanna Winiarsk examining the feasibility of 1,5-AG to monitor glycemic control in patients with T2DM, the concentration of 1,5-AG in well- and poorly controlled patients revealed that individuals with target $\mathrm{HbA} 1 \mathrm{c}$ values might have reduced plasma levels of 1,5-AG [33].

In our study, besides increased postprandial variability, reflected by $1,5-\mathrm{AG}$, the concentration of fasting plasma glucose was increased in both studied groups. More severe hyperglycemia was established among patients with STEMI comparing nonSTEMI. Plasma glucose also was an independent marker that increased the odds of STEMI. In our study, we revealed an association between increased plasma glucose concentration and STEMI odds. However, hyperglycemia in our patients could be not only because of diabetes. The stress-induced hyperglycemia that accompanies the acute phase of myocardial infarction should be considered as a possible confounder. In our study, the adjusted logistic regression model's odds ratio was higher for 1,5-AG than for glucose.

The mechanism of glucosefluctuations is strongly connected with the synthesis of free radicals in human endothelial cells and enhances apoptosis [34]. The episodes of hyperglycemia, reflected by glucose and 1,5-AG can have a prolonged effect on endothelial cells due to epigenetic modifications of the regulatory regions of genes, induce epigenetic modifications of the promoter of the $\mathrm{p} 65$ subunit of Nuclear factor- $\mathrm{\kappa B}$ gene, and increase the expression of inflammatory mediators [35]. Our study establishes decreased 1,5-AG in diabetic patients with STEMI show the presence of postprandial hyperglycemia episodes that probably leads to oxidative stress, endothelial dysfunction, and chronic inflammation, increasing the odds of STEMI. 
The presented study has some limitations because of the relatively small sample size and the characteristics of diabetic patients who were generally with mild decompensation of T2DM. Thus, the results may not be applicable outside of this designation.

In conclusion, we venture to suggest that low $1.5-\mathrm{AG}$ level in blood plasma may be a useful alternative marker of glycemic control outside of $\mathrm{HbA} 1 \mathrm{c}$ and contribute to prevention of macrovascular complications in patients with T2DM.

\section{Conclusion}

Discovered association between 1,5-AG and STEMI in patients with T2DM in our study suggest the potential benefit role of 1,5-AG as a marker of cardiovascular risk in patients with type 2 diabetes mellitus.

Disclosures: There is no conflict of interest for all authors. Acknowledgements: None

Funding: None

\section{References}

1. Einarson TR, Acs A, Ludwig C, Panton UH. Prevalence of cardiovascular disease in type 2 diabetes: a systematic literature review of scientific evidence from across the world in 2007-2017. Cardiovasc Diabetol. 2018;17(1):83. https://doi.org/10.1186/s12933018-0728-6

2. Kannel WB, McGee DL. Diabetes and cardiovascular disease. The Framingham study. JAMA. 1979;241(19):2035-8. https://doi. org/10.1001/jama.1979.03290450033020

3. Stamler J, Vaccaro O, Neaton JD, Wentworth D. Diabetes, other risk factors, and 12-yr cardiovascular mortality for men screened in the Multiple Risk Factor Intervention Trial. Diabetes Care. 1993;16(2):434-444. https://doi.org/10.2337/diacare.16.2.434

4. Aronson D, Rayfield EJ, Chesebro JH. Mechanisms determining course and outcome of diabetic patients who have had acute myocardial infarction. Ann Intern Med. 1997;126(4):296-306. https://doi.org/10.7326/0003-4819-126-4-199702150-00006

5. Sandler CN, McDonnell ME. The role of hemoglobin A1c in the assessment of diabetes and cardiovascular risk. Cleve Clin J Med. 2016;83(5 Suppl 1):S4-S10. https://doi.org/10.3949/ccjm.83.s1.02

6. Fleischer J. Diabetic autonomic imbalance and glycemic variability. J Diabetes Sci Technol. 2012;6(5):1207-1215. https://doi. org/10.1177/193229681200600526

7. Monnier L., Mas E., Ginet C. et al. Activation of oxidative stress by acute glucose fluctuations compared with sustained chronic hyperglycemia in patients with type 2 diabetes. JAMA. 2006;295:1681-1687. https://doi.org/10.1001/jama.295.14.1681

8. Rodbard D. Clinical interpretation of indices of quality of glycemic control and glycemic variability. Postgrad Med. 2011;123(4):107118. https://doi.org/10.3810/pgm.2011.07.2310

9. Chon S., Lee Y.J., Fraterrigo G. et al. Evaluation of glycemic variability in well-controlled type 2 diabetes mellitus. Diabetes Technol Ther. 2013;15(6):455-60. https://doi.org/10.1089/dia.2012.0315

10. Kovatchev B, Cobelli C. Glucose Variability: Timing, Risk Analysis, and Relationship to Hypoglycemia in Diabetes. Diabetes Care. 2016;39(4):502-510. https://doi.org/10.2337/dc15-2035

11. Ceriello A, Colagiuri S. International Diabetes Federation guideline for management of postmeal glucose: a review of recommendations. Diabet Med. 2008;25(10):1151-6. https://doi.org/10.1111/j.1464-5491.2008.02565.x

12. Ma C, Sheng J, Liu Z, Guo M. Excretion rates of 1,5-anhydro-D-glucitol, uric acid and microalbuminuria as glycemic control indexes in patients with type 2 diabetes. Sci Rep. 2017;7:44291. https://doi.org/10.1038/srep44291

13. Klimontov VV, Myakina NE. Glucose variability indices predict the episodes of nocturnal hypoglycemia in elderly type 2 diabetic patients treated with insulin. Diabetes Metab Syndr. 2017;11(2):119-24. https://doi.org/10.1016/j.dsx.2016.08.023

14. Kametani S, Hashimoto Y, Yamanouchi T, Akanuma Y, Akanuma H. Reduced renal reabsorption of 1,5-anhydro-D-glucitol in diabetic rats and mice. J Biochem. 1987;102(6):1599-607. https://doi.org/10.1093/oxfordjournals.jbchem.a122210

15. Yoshioka S., Saitoh S., Negishi C. et al. Variations of 1-deoxyglucose (1,5-anhydroglucitol) content in plasma from patients with insulin-dependent diabetes mellitus. Clin Chem. 1983; 29:1396-1398. https://doi.org/10.1093/clinchem/29.7.1396

16. Yamanouchi T, Minoda S, Yabuuchi M, Akanuma Y, Akanuma H, Miyashita H, et al. Plasma 1,5-anhydro-D-glucitol as new clinical marker of glycemic control in NIDDM patients. Diabetes. 1989;38(6):723-9. https://doi.org/10.2337/diab.38.6.723

17. Yamanouchi T, Tachibana Y, Akanuma H, Minoda S, Shinohara T, Moromizato H, et al. Origin and disposal of 1,5-anhydroglucitol, a major polyol in the human body. Am J Physiol. 1992;263(2 Pt 1):E268-73. https://doi.org/10.1152/ajpendo.1992.263.2.E268

18. 18.Summary of the 2007 European Society of Hypertension (ESH) and European Society of Cardiology (ESC) guidelines for the management of arterial hypertension. Vasc Health Risk Manag. 2007;3(6):783-795.

19. Standards of Medical Care in Diabetes-2018 Abridged for Primary Care Providers. Clin Diabetes. 2018;36(1):14-37. https://doi. org/10.2337/cd17-0119

20. Thygesen K, Alpert JS, Jaffe AS, Simoons ML, Chaitman BR, White HD, et al. Third universal definition of myocardial infarction. Eur Heart J. 2012;33(20):2551-2567. https://doi.org/10.1093/eurheartj/ehs184

21. Dworacka M., Winiarska H. The application of plasma 1,5-anhydro-D-glucitol for monitoring type 2 diabetic patients. Dis Markers. 2005;21(3):127-32. https://doi.org/10.1155/2005/251068

22. Ikeda N., Hara H., Hiroi Y. 1,5-Anhydro-D-glucitol predicts coronary artery disease prevalence and complexity. $J$ Cardiol. 2014;64(4):297-301. https://doi.org/10.1016/j.jjcc.2014.02.014

23. Kuroda M., Shinke T., Sakaguchi K. et al. Effect of daily glucose fluctuation on coronary plaque vulnerability in patients pretreated with lipid-lowering therapy: a prospective observational study. JACC Cardiovasc Interv. 2015;8(6):800-811. https://doi. org/10.1016/j.jcin.2014.11.025

24. Pieber TR, Marso SP, McGuire DK, Zinman B, Poulter NR, Emerson SS, et al. DEVOTE 3: temporal relationships between severe hypoglycaemia, cardiovascular outcomes and mortality. Diabetologia. 2018;61(1):58-65. https://doi.org/10.1007/s00125-017-44220 
25. Yang CD, Shen Y, Ding FH, Yang ZK, Hu J, Shen WF, et al. Visit-to-visit fasting plasma glucose variability is associated with left ventricular adverse remodeling in diabetic patients with STEMI. Cardiovasc Diabetol. 2020;19(1):131. https://doi.org/10.1186/ s12933-020-01112-6

26. Tatti P, Strollo F, Passali D. Sleep apnea, sleep disturbance, and fasting glucose variability: a pilot study. J Diabetes Sci Technol. 2013;7(3):743-8. https://doi.org/10.1177/193229681300700320

27. Hu Y. Advances in reducing cardiovascular risk in the management of patients with type 2 diabetes mellitus. Chronic Dis Transl Med. 2019;5(1):25-36. https://doi.org/10.1016/j.cdtm.2019.01.001

28. Svensson E, Baggesen LM, Johnsen SP, Pedersen L, Nørrelund H, Buhl ES, et al. Early Glycemic Control and Magnitude of HbA1c Reduction Predict Cardiovascular Events and Mortality: Population-Based Cohort Study of 24,752 Metformin Initiators. Diabetes Care. 2017;40(6):800-807. https://doi.org/10.2337/dc16-2271

29. Ghaffari S, Niafar F, Separham A, Niafar M, Pourafkari L, Nader ND. Association between HbA1c levels with severity of coronary artery disease and short-term outcomes of acute ST-elevation myocardial infarction in nondiabetic patients. Ther Adv Cardiovasc Dis. 2015;9(5):305-313. https://doi.org/10.1177/1753944715585500

30. Kranenburg G, van der Graaf Y, van der Leeuw J, Nathoe HM, de Borst GJ, Kappelle LJ, et al. The relation between HbA1c and cardiovascular events in patients with type 2 diabetes with and without vascular disease. Diabetes Care. 2015;38(10):1930-1936. https://doi.org/10.2337/dc15-0493

31. Di Angelantonio E, Gao P, Khan H, Butterworth AS, Wormser D, Kaptoge S, et al. Glycated hemoglobin measurement and prediction of cardiovascular disease. JAMA. 2014;311(12):1225-1233. https://doi.org/10.1001/jama.2014.1873

32. Jarmul JA, Pignone M, Pletcher MJ. Interpreting Hemoglobin A1C in Combination цith conventional risk factors for prediction of cardiovascular risk. Circ Cardiovasc Qual Outcomes. 2015;8(5):501-507. https://doi.org/10.1161/CIRCOUTCOMES.115.001639

33. Dworacka M, Winiarska H. The application of plasma 1,5-anhydro-D-glucitol for monitoring type 2 diabetic patients. Dis Markers. 2005;21(3):127-132. https://doi.org/10.1155/2005/251068

34. Piconi L., Quagliaro L., Assaloni R., et al. Constant and intermittent high glucose enhances endothelial cell apoptosis through mitochondrial superoxide overproduction. Diabetes Metab. Res. Rev. 2006;22(3):198-203. https://doi.org/10.1002/dmrr.613

35. El-Osta A., Brasacchio D., Yao D. et al. Transient high glucose causes persistent epigenetic changes and altered gene expression during subsequent normoglycemia. Journal of Experimental Medicine. 2008;205(10).2409-2417. https://doi.org/10.1084/jem.20081188 